\title{
Low-energy behavior of few-particle scattering amplitudes in two dimensions
}

\author{
Sadhan K. Adhikari \\ Instituto de Física Teórica, Universidade Estadual Paulista, 01405 São Paulo, São Paulo, Brazil \\ William G. Gibson \\ School of Mathematics and Statistics, The University of Sydney, Sydney, New South Wales 2006, Australia
}

(Received 28 May 1992)

\begin{abstract}
A study of the analytic behavior of different few-particle scattering amplitudes at low energies in two space dimensions is presented. Such a study is of use in modeling and understanding different few-particle processes at low energies. A detailed discussion of the energy and the momentum dependence of the partial-wave on-the-energy-shell and off-the-energy-shell two-particle $t$ matrices is given. These $t$-matrix elements tend to zero as the energy and momentum variables tend to zero. The multiple-scattering series is used to show that the connected three-to-three amplitudes diverge in the low-energy-momentum limit. Unitarity relations are used to show that the connected two-tothree and one-to-three amplitudes have specific logarithmic singularities at the $m$-particle breakup threshold. The subenergy singularity in the two-to-three amplitudes is also studied, and comments are made on some applications of the present study in different problems of physical interest.
\end{abstract}

PACS number(s): 03.65.Nk

\section{INTRODUCTION}

Recently, there has been a great deal of activity in the studying and modeling of few-particle problems in two space dimensions, both theoretically [1-6] and experimentally [7-10]. Since it is now possible to experimentally study two-dimensional systems such as helium adsorbed on graphite [10], spin-polarized hydrogen $(\mathrm{H} \downarrow)$ [5,7-9] recombining on a helium film, and multiparticle bound states on monolayers of quantum gases [10], there has been growing interest in the theoretical study of the quantum-mechanical few-particle problem in two dimensions.

Also, in the recent past there has been a number of interesting studies on the quantum and statistical mechanics of anyons [11], which are elementary particles in twospace dimensions having continuous fractional spin and thus interpolating between boson and fermion properties. The possibility of the existence of anyons has increased the relevance of the study of quantum and statistical mechanics in two-space dimensions.

In this paper we present a systematic study of the analytic structures of different few-particle scattering amplitudes in two-space dimensions at low energies, considered as functions of center-of-mass energy and momenta in the initial and final states. A knowledge of such analytic properties at low energies has proved to be of great relevance in the study of quantum-mechanical few-particle problems in three-space dimensions [12]. One cannot usually solve multiparticle-scattering problems exactly; instead, one makes use of simple, tractable models of such processes. Nevertheless, the analytic singularity structure of exact multiparticle amplitudes is of practical interest. It provides guidance in the construction of models of the actual physical processes, because these singularities are often crucial in the reproduction of experimental results. A knowledge of such analytic properties of fewparticle scattering amplitudes in three-space dimensions has revealed the existence of unique and strange effects, such as the Thomas and Efimov effects [12,13]. In twospace dimensions the absence of these effects follows from a consideration of the above-mentioned analytic properties [4].

Although the study of such analytic properties at low energies in two-space dimensions is not expected to directly yield the experimental results, there are implications for physically relevant quantities that one can extract from such study. The low-energy behavior of connected few-particle scattering amplitudes can be used to find the low-temperature behavior of cluster coefficients in quantum-statistical mechanics [14-16]. This could provide a useful check on recent calculations of cluster coefficients for anyons [11]. Such studies are also expected to find applications in other areas in understanding or modeling physical processes in two-space dimensions, an important example being surface recombination of spinpolarized hydrogen atoms.

Reasonably complete discussions of these analytic properties at low energies have appeared for few-particle systems in three-space dimensions [17-20]. Virtually nothing is known about these properties at low energies in two-space dimensions for the $m$-particle system with $m>2$. In the present work, we give a systematic account of these analytic properties in two dimensions for the two- and the three-particle system and provide some general arguments, wherever possible, regarding what to expect for systems involving more particles.

We present a discussion of the low-energy behavior 
of the various two-particle partial-wave $t$-matrix elements, both on-the-energy-shell (on-shell) and off-theenergy-shell (off-shell), in two dimensions. In contrast to the three-dimensional case, the on-shell partial-wave $t$-matrix elements in two dimensions tend to zero as the center of mass (c.m.) energy tends to zero. We relate the off-shell $t$-matrix elements to the on-shell ones and hence predict their low-energy behavior as the energy and momentum variables tend to zero; a significant feature is that the two-particle $t$ matrix has a logarithmic singularity as the c.m. energy tends to zero. We also present a discussion of the effective-range expansion for various partial waves.

Next, we study the low-energy behavior of the connected three-particle scattering amplitude from a consideration of its multiple-scattering (MS) series. Similar use has been made of the MS series to study the analytic properties of the few-particle amplitudes in three dimensions [12]. Such an analysis does not rely on the convergence of the MS series, but only on the very plausible assumption that the singularities present in the individual terms of this series are also present in the full amplitude. As the c.m. energy $E$ of the three-particle system tends to zero, we find that the connected threeto-three scattering amplitude has the divergent behavior given by Eq. (46) below. 'This has consequences for the low-temperature behavior of the third quantum virial coefficient in two dimensions.

We also study the low-energy behavior of the two-totwo and the two-to-three scattering amplitudes of the $m$ particle system, using the unitarity relations satisfied by these amplitudes. Such studies have already appeared for the three-dimensional case [12]. Unlike the connected three-particle scattering amplitude, these amplitudes are finite as the c.m. energy $E$ tends to zero, but they do have branch-cut singularities as $E$ tends to zero. For example, the two-particle scattering amplitude tends to zero at low energies, but it has a logarithmic singularity under these conditions. The two-to-two amplitude develops specific logarithmic singularities at the $m$-particle threshold. The two-to-three amplitude is shown to possess an infinite number of branch points at $E=0$ of the form $\ln ^{-n}(-E), n=1,2,3, \ldots$. Finally, we study the subenergy singularity in the two-to-three scattering amplitude using the subenergy unitarity [12]. This is of interest in the study of the breakup spectrum in two dimensions.

The plan of the paper is as follows. In Sec. II we study the low-energy behavior of the two-particle amplitudes. We present a description of the basic equations, a parametrization of the partial-wave $t$ matrix using phase shifts, a description of unitarity and the optical theorem, and an account of the effective-range expansion and the low-energy behavior of the on-shell $t$ matrix. We also study the off-shell $t$ matrix in some detail, again with emphasis on its low-energy behavior. In Sec. III we study the behavior of three-particle amplitudes, showing that at low energies the connected three-to-three amplitude diverges. In Sec. IV some interesting low-energy singularities in the two-to-two and two-to-three amplitudes are studied. Finally, in Sec. V we present a brief summary.

\section{TWO-PARTICLE AMPLITUDES}

\section{A. Basic equations}

We consider the scattering of a particle of mass $\mu$ in two dimensions by a short-range central potential $v(r)$. We use units where $\hbar^{2} / 2 \mu=1$, and assume that the phase of $E$ is chosen so that $\operatorname{Im} \sqrt{E}>0$. The two-particle $t$ operator at (complex) energy $E$ is defined by the LippmannSchwinger equation

$$
t(E)=v+v g_{0}(E) t(E),
$$

where $g_{0}(E) \equiv\left(E-h_{0}+i 0\right)^{-1}$ is the free Green's function and $h_{0}$ is the free Hamiltonian. In the physical region of interest $E$ is assumed to contain a small positive imaginary part. It is convenient to introduce the wave operator $\omega(E)$ defined by

$$
t(E)=v \omega(E),
$$

which then satisfies

$$
\omega(E)=1+g_{0}(E) v \omega(E) .
$$

We take as a basis the free-particle momentum eigenstates $\mid \mathbf{k}>$ whose space representation is

$$
\langle\mathbf{r} \mid \mathbf{k}\rangle=\frac{1}{2 \pi} \exp (i \mathbf{k} \cdot \mathbf{r})
$$

The free-particle Green's function is then

$$
\left\langle\mathbf{r}^{\prime}\left|g_{0}(E)\right| \mathbf{r}\right\rangle=-\frac{i}{4} H_{0}^{(1)}\left(\left|\mathbf{r}^{\prime}-\mathbf{r}\right| \sqrt{E}\right),
$$

where $H_{0}^{(1)}$ is a Hankel function of the first kind.

We need the following partial-wave expansions $[21,22]$ :

$$
\begin{aligned}
& \langle\mathbf{r} \mid \mathbf{k}\rangle=\frac{1}{2 \pi} \sum_{l=0}^{\infty} \epsilon_{l} i^{l} \cos (l \theta) J_{l}(k r), \\
& \langle\mathbf{r}|\omega(E)| \mathbf{k}\rangle=\frac{1}{2 \pi} \sum_{l=0}^{\infty} \epsilon_{l} i^{l} \cos (l \theta) \omega_{l}(r, k ; E), \\
& \left\langle\mathbf{k}^{\prime}|t(E)| \mathbf{k}\right\rangle=\frac{1}{2 \pi} \sum_{l=0}^{\infty} \epsilon_{l} i^{l} \cos (l \theta) t_{l}\left(k^{\prime}, k ; E\right),
\end{aligned}
$$

$$
\begin{aligned}
& \left\langle\mathbf{r}^{\prime}\left|g_{0}(E)\right| \mathbf{r}\right\rangle \\
& =-\frac{i}{4} \sum_{l=0}^{\infty} \epsilon_{l} \cos (l \theta) J_{l}\left(\sqrt{E} r_{<}\right) H_{l}^{(1)}\left(\sqrt{E} r_{>}\right)
\end{aligned}
$$

where $\epsilon_{l}=2$ for $l \neq 0$ and $\epsilon_{0}=1 ; \theta$ is the angle between the two vectors and $r_{<}\left(r_{>}\right)$is the smaller (larger) of $r$ and $r^{\prime}$. From Eq. (3) we have

$$
\left(-h_{0}+E-v\right) \omega(E)=E-h_{0} \text {. }
$$

If we take a representation of Eq. (10) between $\langle\mathbf{r}|$ and $|\mathbf{k}\rangle$ and expand the relevant quantities in partial waves we obtain the following differential equation for the 
partial-wave components $\omega_{l}(r, k ; E)$ :

$$
\begin{aligned}
{\left[\frac{1}{r} \frac{\partial}{\partial r}\left(r \frac{\partial}{\partial r}\right)-\frac{l^{2}}{r^{2}}+E-v(r)\right] } & \omega_{l}(r, k ; E) \\
& =\left(E-k^{2}\right) J_{l}(k r) .
\end{aligned}
$$

This equation shows that $\omega_{l}(r, k ; E)$ can be looked upon as an off-shell radial wave function $[17,18]$, with $\omega_{l}\left(r, k ; k^{2}\right)$ just the usual radial wave function. From Eq. (3) one can also obtain an integral representation for $\omega_{l}(r, k ; E)$,

$$
\begin{aligned}
\omega_{l}(r, k ; E)=J_{l}(k r)-\frac{\pi i}{2} \int_{0}^{\infty} & r^{\prime} d r^{\prime} J_{l}\left(\sqrt{E} r_{>}\right) \\
& \times H_{l}^{(1)}\left(\sqrt{E} r_{>}\right) t_{l}\left(r^{\prime}, k ; E\right) .
\end{aligned}
$$

Examining the form of Eq. (12) as $r \rightarrow \infty$, and using the relation

$$
t_{l}\left(k^{\prime}, k ; E\right)=\int_{0}^{\infty} r^{\prime} d r^{\prime} J_{l}\left(k^{\prime} r^{\prime}\right) t_{l}\left(r^{\prime}, k ; E\right),
$$

we obtain

$\omega_{l}(r, k ; E) \sim J_{l}(k r)-\frac{\pi i}{2} H_{l}^{(1)}(\sqrt{E} r) t_{l}(\sqrt{E}, k ; E)$

as $r \rightarrow \infty$.

If we take the radial wave function to have the standard asymptotic form $[17,22]$

$$
\omega_{l}\left(r, k, k^{2}\right) \sim \frac{2}{\pi k r} e^{i \delta_{l}} \cos \left[k r-\frac{\pi}{2}\left(l+\frac{1}{2}\right)+\delta_{l}\right]
$$

as $r \rightarrow \infty$,

where $\delta_{l} \equiv \delta_{l}(k)$ is the phase shift for the $l$ th partial wave, then Eq. (14) can be used to show that the on-shell $t$ matrix element has the following expression in terms of the phase shift:

$$
t_{l}\left(k, k ; k^{2}\right)=-\frac{2}{\pi} e^{i \delta_{l}} \sin \delta_{l} .
$$

This expression for the partial-wave $t$ matrix satisfies the following on-shell unitarity relation

$$
\operatorname{Im} t_{l}\left(k, k ; k^{2}\right)=-\frac{\pi}{2}\left|t_{l}\left(k, k ; k^{2}\right)\right|^{2}
$$

A complete account of off-shell unitarity relations for the partial-wave $t$ matrix and the relation of this partialwave $t$ matrix to the physical scattering amplitude has appeared elsewhere [21].

\section{B. On-shell behavior}

We are interested in the low-energy behavior of scattering quantities, and start by investigating their on-shell behavior. The basic quantities are the phase shifts $\delta_{l}(k)$, and their form at low energies has been the subject of a number of recent investigations [23-25]. A fundamental result, first emphasized by Bolle and Gesztesy [23], is that the effective-range expression

$$
k^{2 l}\left[(\pi / 2) \cot \delta_{l}-\ln k\right]
$$

has an expansion in powers of $k^{2}$ which is convergent or asymptotic, depending on the potential. The noteworthy feature is the occurrence of the logarithmic term in Eq. (18). In three dimensions this logarithmic term is absent and the effective-range expression is $k^{l} \cot \delta_{l}$. In fact, such a logarithmic term is present only for even space dimensions $(2,4, \ldots)$. In terms of the energy $E=k^{2}$, we have [21]

$$
\cot \delta_{l}=\mathcal{A}_{l}(E)+(1 / \pi) \ln E,
$$

where $\mathcal{A}_{l}(E)$ contains no logarithmic terms, and possesses an expansion

$$
\mathcal{A}_{l}(E)=\alpha_{l} E^{-l}+\alpha_{l-1} E^{-l+1}+\cdots .
$$

This enables us to find low-energy expansions for the onshell $t$-matrix elements. Rewriting Eq. (16) in the form

$$
t_{l}(E) \equiv t_{l}\left(k, k ; k^{2}\right)=-(2 / \pi)\left(\cot \delta_{l}-i\right)^{-1},
$$

we have, from Eq. (19),

$$
t_{l}(E)=-(2 / \pi)\left[\mathcal{A}_{l}(E)+(1 / \pi) \ln (-E)\right]^{-1} .
$$

Since the analytic continuation of $t_{l}(E)$ to real negative energies must be real, this relation shows that $\mathcal{A}_{l}(E)$ is a real function of $E$.

The low-energy expansion of the $t$ matrix can be found from Eqs. (20) and (22). We now investigate this in some detail. There are two points of particular interest. First, the expansion is qualitatively different for $l=0$, where the $\ln (-E)$ term dominates, as compared to $l \geq$ 1 , where $E^{l}$ dominates. Second, the leading coefficients in this expansion can be given a physical interpretation, analogous to the familiar scattering length and effective range of three-dimensional scattering.

We first consider the $l=0$ ( $s$-wave) case. A possible parametrization is

$$
\frac{\pi}{2} \cot \delta_{0}-\left(\gamma+\ln \frac{k}{2}\right)=-\frac{1}{a_{0}}+\frac{1}{2} r_{0} k^{2}+\cdots,
$$

with $a_{0}$ the "scattering length" and $r_{0}$ the "effective range." The constant $\gamma=0.57721 \ldots$ is Euler's constant. We note that other parametrizations are possible [23-25] and, in particular, that in practical applications there is good reason to introduce a further quantity $R$ so that the logarithmic term becomes $\ln (R k / 2)$ and $a_{0}$ is correspondingly modified [25]. Because of the presence of the logarithmic term in Eq. (23) the numerical value of the scattering length, $a_{0}$, depends on the dimension of en$\operatorname{ergy} E$ or of momentum $k$. In three dimensions this logarithmic term is absent and in the usual effective-range expansion, 


$$
k \cot \delta_{0}=-\frac{1}{a_{0}}+\frac{1}{2} r_{0} k^{2}
$$

the numerical value of the scattering length $a_{0}$ does not depend on the dimension of momentum employed. Thus there is an inherent uncertainty in defining the scattering length in two dimensions.

If we use Eq. (23) in Eq. (21) we obtain the expansion

$$
\begin{aligned}
t_{0}(E)= & {\left[-\frac{2}{\ln (-E)}+\frac{2 b}{[\ln (-E)]^{2}}-\frac{2 b^{2}}{[\ln (-E)]^{3}}+\cdots\right] } \\
& +\frac{2 r_{0} E}{[\ln (-E)]^{2}}-\frac{4 b r_{0} E}{[\ln (-E)]^{3}}+O\left(\frac{E}{\ln (-E)]^{4}}\right),
\end{aligned}
$$

where $b=2\left(\gamma-\ln 2-1 / a_{0}\right)$. As $E \rightarrow 0$ only the terms in the first bracket, which is simply the expansion of $-2[\ln (-E)+b]^{-1}$, are important. However, for $E$ not too small some of the other terms, which are all of the form $E^{i} /[\ln (-E)]^{n}$ with $i \geq 1$ and $n \geq 2$, may be important. Note that $t_{0}(E) \rightarrow 0$ as $E \rightarrow 0$; this is to be contrasted with the three-dimensional case where the zero-energy $s$-wave $t$ matrix is proportional to the scattering length.

For the $l=1$ partial wave ( $p$ wave) the phase shift at low energies can be parametrized as

$k^{2}\left[\frac{\pi}{2} \cot \delta_{1}-\left(\gamma+\ln \frac{k}{2}\right)\right]=-\frac{1}{a_{1}}+\frac{1}{2} r_{1} k^{2}+\cdots$,

where $a_{1}$ and $r_{1}$ are the $p$-wave scattering length and effective range, respectively. Equation (26) yields the following low-energy expansion for the $p$-wave $t$ matrix:

$t_{1}(E)=a_{1} E+\frac{1}{2} a_{1}^{2} E^{2} \ln (-E)+a_{1}^{2} c E^{2}+O\left(E^{3}[\ln (-E)]^{2}\right)$, where $c=\gamma-\ln 2+\frac{1}{2} r_{1}$. For small $E$ these terms are much smaller than the leading terms in the expansion of the $s$-wave amplitude given in Eq. (25).

For general $l \geq 1$ the leading term in the expansion of $t_{l}(E)$ will involve $E^{l}$, and the first logarithmic term will enter as $E^{2 l} \ln (-E)$; that is, the form of the low-energy expansion is

$$
t_{l}(E)=\beta_{l} E^{l}+\beta_{l+1} E^{l+1}+\cdots+\gamma_{2 l} E^{2 l} \ln (-E)+\cdots .
$$

\section{Off-shell behavior}

In order to study three- and more-particle effects we may need the two-particle $t$ matrix off the energy shell. In particular, we want the low-energy behavior of the fully off-shell $t$-matrix elements $t_{l}\left(k^{\prime}, k ; E\right)$, where $k^{\prime}, k$ are arbitrary real quantities, and, as usual, $E$ is considered to be complex with $\operatorname{Im} \sqrt{E}>0$. We start by deriving a general relation between this quantity and the half-off-shell $t$ matrix, $t_{l}(\sqrt{E}, k ; E)$. Similar relations have appeared in the three-dimensional case $[15,18]$.

Let us start by defining the quantity

$\bar{\omega}_{l}(r, k ; E)=J_{l}(k r)-\frac{\pi i}{2} H_{l}^{(1)}(\sqrt{E} r) t_{l}(\sqrt{E}, k ; E)$.

From Eq. (14) we see that $\bar{\omega}_{l}(r, k ; E)$ is the asymptotic form of the off-shell wave function $\omega_{l}(r, k ; E)$. From Eqs. (2) and (3) we have

$$
t(E)=\left[g_{0}(E)\right]^{-1}[\omega(E)-1]
$$

If we consider the matrix representation of Eq. (30) between the plane-wave states $\left\langle\mathbf{k}^{\prime}\right|$ and $|\mathbf{k}\rangle$, we obtain

$$
\left\langle\mathbf{k}^{\prime}|t(E)| \mathbf{k}\right\rangle=\left(E-k^{\prime 2}\right) \int d^{2} r\left\langle\mathbf{k}^{\prime} \mid \mathbf{r}\right\rangle[\langle\mathbf{r}|\omega(E)| \mathbf{k}\rangle-\langle\mathbf{r} \mid \mathbf{k}\rangle] .
$$

If we use the partial-wave expansions, Eqs. (6)-(9), in Eq. (31) we immediately obtain the following integral representation of the off-shell partial-wave $t$-matrix elements:

$$
t_{l}\left(k^{\prime}, k ; E\right)=\left(E-k^{\prime 2}\right) \int_{0}^{\infty} r d r J_{l}\left(k^{\prime} r\right)\left[\omega_{l}(r, k ; E)-J_{l}(k r)\right] .
$$

Using Eq. (29), if we substitute for $J_{l}(k r)$ in terms of $\bar{\omega}_{l}$, Eq. (32) can be rewritten as

$$
\begin{aligned}
t_{l}\left(k^{\prime}, k ; E\right)= & \left(E-{k^{\prime}}^{2}\right) \int_{0}^{\infty} r d r J_{l}\left(k^{\prime} r\right)\left[\omega_{l}(r, k ; E)-\bar{\omega}_{l}(r, k ; E)\right] \\
& -\left(E-k^{\prime 2}\right) \int_{0}^{\infty} r d r J_{l}\left(k^{\prime} r\right) \frac{\pi i}{2} H_{l}^{(1)}(\sqrt{E} r) t_{l}(\sqrt{E}, k ; E) .
\end{aligned}
$$

The second of the integrals in this equation can be evaluated using the following standard result:

$$
\int_{0}^{\infty} r d r J_{l}(p r) H_{l}^{(1)}(s r)=-\frac{2 i}{\pi} \frac{1}{p^{2}-s^{2}}\left(\frac{p}{s}\right)^{l},
$$

which is valid for $l \geq 0$ and $\operatorname{Im} s>0$. Using Eq. (34), Eq. (33) can be rewritten as

$$
t_{l}\left(k^{\prime}, k ; E\right)=\left(\frac{k^{\prime}}{\sqrt{E}}\right)^{l} t_{l}(\sqrt{E}, k ; E)+\left(E-{k^{\prime}}^{2}\right) \int_{0}^{\infty} r d r J_{l}\left(k^{\prime} r\right)\left[\omega_{l}(r, k ; E)-\bar{\omega}_{l}(r, k ; E)\right]
$$


This is the required relation between the fully-off-shell and the half-off-shell $t$-matrix elements. Using the partial-wave expansions Eqs. (6)-(8) in the fully off-shell matrix element of Eq. (2) we obtain the following relation, after some straightforward algebra:

$$
t_{l}\left(k^{\prime}, k ; E\right)=\int_{0}^{\infty} r d r v(r) J_{l}\left(k^{\prime} r\right) \omega_{l}(r, k ; E) .
$$

If we put $k=\sqrt{E}$ in Eq. (35), we obtain the following relation between the half-off-shell $t$-matrix elements and the on-shell $t$-matrix elements:

$$
t_{l}\left(k^{\prime}, \sqrt{E} ; E\right)=\left(\frac{k^{\prime}}{\sqrt{E}}\right)^{l} t_{l}(E)+\left(E-{k^{\prime}}^{2}\right) \int_{0}^{\infty} r d r J_{l}\left(k^{\prime} r\right)\left[\omega_{l}(r, \sqrt{E} ; E)-\bar{\omega}_{l}(r, \sqrt{E} ; E)\right] .
$$

Here, $\omega_{l}(r, \sqrt{E} ; E)$ is just the usual radial wave function with asymptotic form given by Eq. (15).

We now wish to investigate the behavior of $t_{l}\left(k^{\prime}, k ; E\right)$ as all the quantities $k^{\prime}, k$, and $E$ tend to zero. Again, the $s$-wave case turns out to be somewhat different from the $l \geq 1$ cases, and first we treat this case separately. We start by considering Eq. (37) as $E \rightarrow 0$. It follows from Eqs. (12) and (29) that both $\omega_{0}(r, \sqrt{E} ; E)$ and $\bar{\omega}_{0}(r, \sqrt{E} ; E)$ behave like $(\ln E)^{-1}$ as $E \rightarrow 0$. This leads us to define

$\chi_{0}=\lim _{E \rightarrow 0} \int_{0}^{\infty} r d r \ln (-E)\left[\omega_{0}(r, \sqrt{E} ; E)-\bar{\omega}_{0}(r, \sqrt{E} ; E)\right]$

Then taking the low-energy-momentum limit of Eq. (37) we obtain

$$
\begin{aligned}
t_{0}\left(k^{\prime}, \sqrt{E} ; E\right) \sim t_{0}(E)+\left(E-k^{\prime 2}\right) & \frac{\chi_{0}}{\ln (-E)} \\
& \text { as } k^{\prime}, E \rightarrow 0 .
\end{aligned}
$$

Similarly, the low-energy-momentum limit of Eq. (35) yields

$$
\begin{array}{r}
t_{0}\left(k^{\prime}, k ; E\right) \sim t_{0}(\sqrt{E}, k ; E)+\left(E-{k^{\prime}}^{2}\right) \frac{\chi_{0}}{\ln (-E)} \\
\text { as } k, k^{\prime}, E \rightarrow 0 .
\end{array}
$$

From Eqs. (39) and (40) we obtain

$$
\begin{aligned}
t_{0}\left(k^{\prime}, k ; E\right) \sim t_{0}(E)+\frac{\left(2 E-k^{2}-k^{2}\right) \chi_{0}}{\ln (-E)} & \text { as } k, k^{\prime}, E \rightarrow 0 .
\end{aligned}
$$

The expansion given in Eq. (25) for $t_{0}(E)$ then gives

$$
t_{0}\left(k^{\prime}, k ; E\right)=\left[-\frac{2}{\ln (-E)}+\frac{2 b}{[\ln (-E)]^{2}}-\frac{2 b^{2}}{[\ln (-E)]^{3}}+\cdots\right]+\frac{\left(2 E-k^{2}-k^{2}\right) \chi_{0}}{\ln (-E)}+\cdots
$$

It is interesting to note that the off-shell parameter $\chi_{0}$ occurs before the effective-range parameter $r_{0}$-the latter only enters with the $E /[\ln (-E)]^{2}$ term. The constant $\chi_{0}$ can be calculated given the potential $v(r)$; for example, for hard disks of diameter $a$ it is $-a^{2} / 2$.

A similar analysis can be performed for $l \geq 1$. In this case, the appropriate off-shell parameter is

$$
\begin{array}{r}
\chi_{l}=\lim \frac{1}{2^{l} l !} \int_{0}^{\infty} r^{l+1} d r k^{-l}\left[\omega_{l}(r, k ; E)-\bar{\omega}_{l}(r, k ; E)\right] \\
\text { as } k, E \rightarrow 0 .
\end{array}
$$

Then, following the exactly same steps used to derive Eq. (42), we obtain for $l=1$,

$$
\begin{aligned}
t_{1}\left(k^{\prime}, k ; E\right)=k^{\prime} k[ & a_{1}+\frac{1}{2} a_{1}^{2} E \ln (-E)+a_{1}^{2} c E \\
& \left.+\left(2 E-k^{2}-k^{2}\right) \chi_{1}+\cdots\right],
\end{aligned}
$$

and for $l \geq 2$,

$t_{l}\left(k^{\prime}, k ; E\right)=\left(k^{\prime} k\right)^{l}\left[\beta_{l}+\beta_{l+1} E+\left(2 E-k^{\prime 2}-k^{2}\right) \chi_{l}+\cdots\right]$.

\section{THREE-TO-THREE AMPLITUDES}

In this section we shall discuss the low-energy behavior of the connected three-particle scattering amplitude. Such a study is important for investigating the lowtemperature behavior of quantum cluster coefficients, as we have emphasized in the Introduction. We shall in particular show that as the three-particle center-of-mass energy $E$ tends to zero, the on-shell connected threeparticle amplitude $T_{3 \rightarrow 3}$ diverges like

$T_{3 \rightarrow 3} \sim E^{-1}\left(\frac{A}{\ln ^{2}(-E)}+\frac{B}{\ln ^{3}(-E)}+\frac{C}{\ln ^{4}(-E)}+\ldots\right)$,

where $A, B, C, \ldots$ are constants which are completely determined by the low-energy behavior of the on-shell two-particle $s$-wave amplitude $t_{0}(E)$ which we studied in Sec. II. We present a systematic way of calculating the coefficients of all the divergent terms of Eq. (46).

As in Refs. [12] and [19] we find the divergent parts of the connected three-particle amplitude $T_{3 \rightarrow 3}$ from a study of the lower-order diagrams of the multiple scat- 


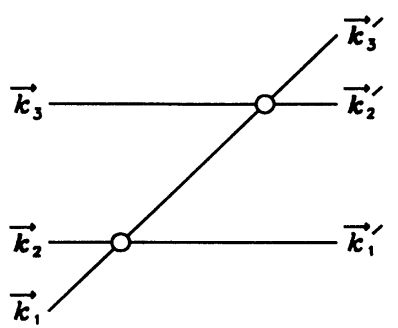

(a)

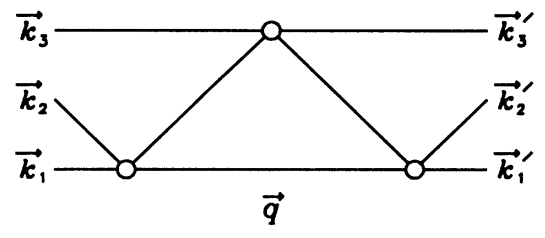

(b)

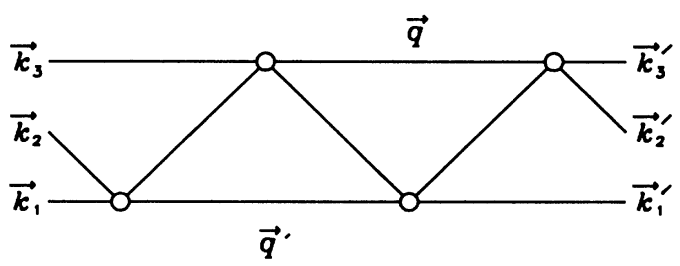

(c)

FIG. 1. Typical (a) second-, (b) third-, and (c) fourthorder MS graphs in the c.m. frame. The circles indicate two-particle $t$ matrices.

tering (MS) series, as shown in Fig. 1. Although we base our study on the MS series, our conclusion does not rely on the convergence properties of this series. (In fact, at low energies this series is usually a divergent one.)
There cannot be, in general, a cancellation between the various terms and hence the analytic properties obtained from the study of a specific term of the MS series will be present in the full physical amplitude.

We study the case of three particles with momenta $\mathbf{k}_{1}, \mathbf{k}_{2}, \mathbf{k}_{3}$ in the c.m. frame going to momenta $\mathbf{k}_{1}^{\prime}, \mathbf{k}_{2}^{\prime}, \mathbf{k}_{3}^{\prime}$ after elastic scattering. The particles are considered to have mass $\mu$ and we use units $\hbar=2 \mu=1$ throughout the rest of the paper. Though the particles are considered to be of equal mass, we assume that they are distinguishable. These restrictions are imposed to simplify the discussion, which can easily be extended to other cases. We have for the total c.m. energy

$$
E=\sum_{i=1}^{3} k_{i}^{2}=\sum_{i=1}^{3}{k^{\prime}}^{2}
$$

Also, our working in the c.m. system implies

$$
\sum_{i=1}^{3} \mathbf{k}_{i}=\sum_{i=1}^{3} \mathbf{k}_{i}^{\prime}=\mathbf{0} .
$$

It is advantageous to work in terms of dimensionless momentum variables $\mathbf{y}_{i}$ and $\mathbf{y}_{i}^{\prime}$ defined by

$$
\mathbf{k}_{i}=\sqrt{E} \mathbf{y}_{i}, \quad k_{i}^{\prime}=\sqrt{E} \mathbf{y}_{i}^{\prime}, \quad i=1,2,3
$$

We shall assume that at low energies $\mathbf{k}, \mathbf{k}^{\prime}$, etc. go to zero linearly with $\sqrt{E}$.

The lower order diagrams in the MS series, as shown in Fig. 1, will contribute to the divergent terms in Eq. (46). The contribution of Fig. 1(a), a typical secondorder rescattering diagram, expressed in terms of $y^{\prime} s$, is

$$
\frac{\left\langle\frac{1}{2} \sqrt{E}\left(\mathbf{y}_{1}-\mathbf{y}_{2}\right)\left|t_{12}\left[E\left(1-\frac{3}{2} y_{3}^{2}\right)\right]\right| \sqrt{E}\left(\mathbf{y}_{1}^{\prime}+\frac{1}{2} \mathbf{y}_{3}\right)\right\rangle}{E\left[1-y_{1}^{\prime 2}-y_{3}^{2}-\left(\mathbf{y}_{1}^{\prime}+\mathbf{y}_{3}\right)^{2}\right]}\left\langle\sqrt{E}\left(\mathbf{y}_{3}+\frac{1}{2} \mathbf{y}_{1}^{\prime}\right)\left|t_{23}\left[E\left(1-\frac{3}{2} y_{1}^{\prime 2}\right)\right]\right| \frac{1}{2} \sqrt{E}\left(\mathbf{y}_{3}^{\prime}-\mathbf{y}_{2}^{\prime}\right)\right\rangle,
$$

where $\left\langle\mathbf{k}\left|t_{i j}(\epsilon)\right| \mathbf{k}^{\prime}\right\rangle$ is the off-shell two-particle $t$ matrix of the pair $i j$ from relative momentum $\mathbf{k}$ to relative momentum $\mathbf{k}^{\prime}$ at c.m. energy $\epsilon$. At low energies the off-shell two-particle $t$ matrices $t_{12}$ and $t_{23}$ tend to zero, as we have seen in Sec. II. But, as the leading low-energy behavior of $t_{12}$ and $t_{23}$ is of the form $\ln ^{-n}(-E), n \geq 1$, at low energies $(E \rightarrow 0)$ the expression (50) diverges because of its energy denominator. It is easy to see that expression (50) will contribute to all the constants $A, B, C, \ldots$, of Eq. (46). We shall not attempt to calculate the contribution of Eq. (50) to various coefficients in Eq. (46). But it is interesting to note that these contributions can be calculated from the on-shell behavior of the two-particle $t$ matrix. The lowest-order correction to the off-shell twoparticle $t$ matrix, as we have found in Sec. II, will not contribute to the divergent terms of Eq. (46). We should also remember that we shall have to consider all possible permutations of particles in expression (50). Though we shall not explicitly carry out such permutations in this paper, they will be implied.

Next, let us consider the typical third-order rescattering diagram of Fig. 1(b), whose contribution to the connected three-particle amplitude is given by

$$
\frac{1}{(2 \pi)^{2}} \int d \mathbf{q} \frac{t_{12}\left[E\left(1-\frac{3}{2} y_{3}^{2}\right)\right] t_{23}\left(E-\frac{3}{2} q^{2}\right) t_{12}\left[E\left(1-\frac{3}{2}{y_{3}^{\prime}}_{3}^{2}\right)\right]}{\left(E-2 k_{3}^{2}-2 q^{2}-2 \mathbf{k}_{3} \cdot \mathbf{q}\right)\left(E-2 k_{3}^{\prime 2}-2 q^{2}-2 \mathbf{k}_{3}^{\prime} \cdot \mathbf{q}\right)}
$$


The momentum levels of the two-particle $t$ matrices in expression (51) are suppressed to save space. As it is reasonable to assume that for a well-behaved short-range potential the middle $t$ matrix $t_{23}$ decays sufficiently rapidly to zero for large $q$, the integral (51) is perfectly finite at the upper limit. So in order to find the divergent part of integral (51) as $E \rightarrow 0$ we introduce a cutoff parameter $\Lambda$ in $q$. In order to get the most divergent part of integral (51) we employ the lowest-order terms of the two-particle $t$ matrices, given by Eq. (25), in Eq. (51) and we get as $E \rightarrow 0$,

$$
\frac{-8}{(2 \pi)^{2} E \ln ^{2}(-E)} \int d \Omega \int_{0}^{\Lambda / \sqrt{E}} x d x \frac{1}{\left(1-2 y_{3}^{2}-2 x^{2}-2 \mathbf{y}_{3} \cdot \mathbf{x}\right)} \frac{1}{\left(1-2 y_{3}^{\prime 2}-2 x^{2}-2 \mathbf{y}_{3}^{\prime} \cdot \mathbf{x}\right) \ln \left[\left(\frac{3}{2} x^{2}-1\right) E\right]}
$$

where $d \Omega$ represents integrations over angles of $\mathbf{x}$ defined by $\mathbf{x} \sqrt{E}=\mathbf{q}$. As $E \rightarrow 0$ and $x \rightarrow \Lambda / \sqrt{E}$, the $\left\{\ln \left[\left(\frac{3}{2} x^{2}-1\right) E\right]\right\}^{-1}$ term of (52) tends to zero as $\Lambda \rightarrow 0$, and expression (52) diverges like $E^{-1} \ln ^{-2}(-E)$, as the remaining $x$ integral remains finite as $E \rightarrow 0$.

Now, it is easy to realize that if we had kept higherorder terms in the low-energy expansion of the twoparticle $t$ matrices in expression (51) we would have found terms divergent as $E^{-1} \ln ^{-n}(-E), n \geq 2$ as $E \rightarrow 0$, which would have contributed to the coefficients of different terms in expression (46). It is also clear that the higher order terms in the low-energy expansion of the offshell $t$ matrix-the terms which are not contained in the first square bracket of Eq. (42)-when substituted in expression (51) will not lead to any divergent terms. So the third-order rescattering diagram of Fig. 1(b) contributes to coefficients of all the divergent terms of expression (46).

Next, the contribution of Fig. 1(c), a typical fourthorder rescattering diagram, to the connected threeparticle scattering amplitude is

$$
\frac{1}{(2 \pi)^{4}} \iint d \mathbf{q} d \mathbf{q}^{\prime} \frac{t_{12} t_{23} t_{12} t_{31}}{\left(E-2 k_{3}^{2}-2 q^{2}-2 \mathbf{k}_{3} \cdot \mathbf{q}\right)\left(E-2 q^{2}-2{q^{\prime}}^{2}-2 \mathbf{q} \cdot \mathbf{q}^{\prime}\right)} \frac{1}{\left(E-2{q^{\prime}}^{2}-2{k^{\prime}}^{2}-2 \mathbf{q} \cdot \mathbf{k}_{1}^{\prime}\right)},
$$

where both the energy and momentum variables of the $t$ matrices have again been suppressed to save space. As in the case of the third order rescattering diagram, the most divergent part of expression (53) can be calculated by considering the lowest-order terms in the two-particle $t$ matrices in expression (53). As before, both the $\mathbf{q}$ and $\mathbf{q}^{\prime}$ integrations will be finite at the upper limit and one can introduce a cutoff $\Lambda$ in $\mathbf{q}$ and $\mathbf{q}^{\prime}$ as $E \rightarrow 0$. The most divergent part of expression (53) as $E \rightarrow 0$ can be obtained by considering the lowest-order terms of the two-particle $t$ matrices and separating the radial and angular integrals as follows:

$$
\begin{aligned}
\frac{16}{(2 \pi)^{4} E \ln ^{2}(-E)} \int_{\Omega_{x}} d \Omega_{x} \int_{\Omega_{y}} d \Omega_{y} \int_{0}^{x=y=\Lambda / \sqrt{E}} x d x & y d y \frac{1}{\left(1-2 y_{3}^{2}-2 x^{2}-2 \mathbf{y}_{3} \cdot \mathbf{x}\right)\left(1-2 x^{2}-2 y^{2}-2 \mathbf{x} \cdot \mathbf{y}\right)} \\
& \times \frac{1}{\left(1-2 y^{2}-2{y^{\prime}}_{1}^{2}-2 \mathbf{y} \cdot \mathbf{y}_{1}^{\prime}\right) \ln \left[\left(\frac{3}{2} x^{2}-1\right) E\right] \ln \left[\left(\frac{3}{2} y^{2}-1\right) E\right]}
\end{aligned}
$$

where $d \Omega_{x}$ and $d \Omega_{y}$ represent integrations over the angles of $\mathbf{x}$ and $\mathbf{y}$ defined by $\mathbf{x} \sqrt{E}=\mathbf{q}, \mathbf{y} \sqrt{E}=\mathbf{q}^{\prime}$, respectively. As before, we shall be concerned with the upper limit of $x$ and $y$ integrations as $E \rightarrow 0$. Again the logarithmic terms $\ln ^{-1}\left[\left(\frac{3}{2} x^{2}-1\right) E\right]$ and $\ln ^{-1}\left[\left(\frac{3}{2} y^{2}-1\right) E\right]$ under the integral sign in expression (54) remain finite as $E \rightarrow 0$ and $x, y \rightarrow \Lambda / \sqrt{E}$. Hence, in the appropriate limit as $E \rightarrow 0$, the whole integral in expression (54) is finite and this expression diverges as $E^{-1} \ln ^{-2}(-E)$. It is now easy to realize that if we had kept higher-order terms in the low-energy expansions of the two-particle $t$ matrices in expression (53), we would have found terms divergent as $E^{-1} \ln ^{-n}(-E), n \geq 2$ as $E \rightarrow 0$ which would have contributed to the coefficients of different terms in Eq. (46). As in Eq. (51), if we keep the off-shell terms of Eq. (42) in the two middle $t$ matrices of expression (53), we would find that this expression does not lead to any divergent term at low energies.
In a similar way one can study the connected $n$th order $(n>3)$ three-particle rescattering diagrams at low energies. As we increase the order of multiple scattering by one, we introduce one integration over an intermediate momentum, one energy denominator, and a twoparticle $t$ matrix, which is clear from expressions (51) and (53), when we go from second- to third-order rescattering. By dimensional argument the two-dimensional integration compensates for the energy denominator and no new type of divergent term emerges as the new twoparticle $t$ matrix does not diverge under appropriate limits. Hence each of the $n$ th-order three-particle rescattering diagrams behaves as $E^{-1} \ln ^{-n}(-E), n \geq 2$ as $E \rightarrow 0$.

This rule seems to be true in general. The connected $m$-particle scattering amplitude will also diverge as the $m$-particle c.m. energy $E \rightarrow 0$. From a dimensional argument it can be realized that the divergent terms will behave as $E^{-(m-2)} \ln ^{-n}(-E), n \geq 2$ as $E \rightarrow 0$. The 
coefficients of these divergent terms can be calculated, in principle, from the leading low-energy behavior of the on-shell two-particle $t$ matrix in two dimensions.

The divergent behavior of the connected three-particle amplitude was conjectured by Gibson [16] in his study of quantum cluster coefficients in two dimensions.

We have seen before $[12,19]$ that in three dimensions also the connected few-particle amplitude diverges as the c.m. energy $E \rightarrow 0$. This behavior was crucial in predicting the low-temperature behavior of quantum cluster coefficients. There are two crucial differences between the low-energy behavior of few-particle scattering amplitudes in two and three dimensions. First, in three dimensions the higher-order rescattering diagrams may lead to new types of divergent terms not contained in the lowest-order term. Second, in three dimensions there are only a finite number of lower-order rescattering diagrams that diverge at low energies. In two-dimensional systems these two properties do not hold. In two dimensions consideration of higher-order rescattering diagrams does not lead, at low energies, to new types of singularities which are not contained in the lowest-order diagram. Also, in two dimensions all the terms in the $m$-particle multiplescattering series diverge at low energies for $m>2$. This latter fact will make any attempt to calculate the coefficients of the series (46), for example, practically impossible, though such a calculation is possible in principle from a knowledge of the leading low-energy behavior of the two-particle amplitude.

\section{TWO-TO-TWO AND TWO-TO-THREE AMPLITUDES}

\section{A. Introductory remarks}

In view of the recent studies [1-10] of exact threeparticle calculations and of the effect of three-particle correlations in surface three-particle recombination in spin-polarized atomic hydrogen [5], it is interesting to study the singularity structure of other multiparticle amplitudes in two dimensions. A knowledge of this singularity structure is essential for a correct parametrization of these amplitudes and this is important for an approximate treatment of three-particle processes.

One important question is what is the behavior of a two-to-two amplitude at the $m$-particle breakup threshold in two dimensions. The simplest relevant threshold is the three-particle breakup threshold. The process we are addressing is of the general type

$$
i+(j k) \rightarrow k+(i j)
$$

where $(j k)$ represents the bound state of particles $j$ and $k, i \neq j \neq k \neq i$. Here each of $i, j, k$ runs from 1 to 3 . At the breakup threshold the "two-particle" amplitude described above develops a particular singularity, which we shall study in Sec. IV B.

Other important amplitudes of interest are the two-tothree and the one-to-three amplitudes and their singularity behaviors at the three-particle threshold. Of these, the one-to-three amplitude is of particular interest as it is the time-reversed form of the three-to-one amplitude which describes the three-particle recombination process in spin-polarized atomic hydrogen. Again, these analytic behaviors are important for a correct parametrization of these amplitudes. We shall study this singularity in Sec. IV C.

An analysis similar to that of Sec. III reveals that both the two-to-two and the two-to-three amplitudes approach constants in the low-energy limit. In order to see this, we consider the lowest-order terms of the MS series, as shown in Figs. 2(a) and 2(b), for the two-to-two and the two-to-three scattering amplitudes of the three-particle system. Apart from the vertex functions and two-particle $t$ matrix elements, both of which tend to constants as $E \rightarrow 0$, these two terms contain the energy denominator

$$
\left[E-k^{2}-{k^{\prime}}_{1}^{2}-\left(\mathbf{k}+\mathbf{k}_{1}^{\prime}\right)^{2}\right]^{-1}
$$

as in Eq. (50). For the two-to-two scattering amplitude, the energy-conservation condition is now

$$
E+\alpha^{2}=\frac{3}{2} k^{2}=\frac{3}{2} k_{1}^{\prime 2},
$$

where $\alpha^{2}$ is the two-particle binding energy, while for the two-to-three scattering amplitude we have

$$
E+\alpha^{2}=\frac{3}{2} k^{2}
$$

and

$$
E={k^{\prime}}_{1}^{2}+{k^{\prime}}_{2}^{2}+{k^{\prime}}_{3}^{2} \text {. }
$$

With these conditions we see that, unlike Eq. (50), the energy denominator above does not necessarily vanish as $E \rightarrow 0$, because $k^{2}$ tends to a nonvanishing constant unless $\alpha^{2}$ is zero. Hence the amplitudes of Fig. 2 do not diverge as $E \rightarrow 0$, nor do the corresponding higher-order diagrams.

Although these amplitudes are finite, they have a branch-cut singularity as $E \rightarrow 0$ arising from the scattering threshold. For example, the two-particle $t$ matrix is finite as $E \rightarrow 0$, but it does have a logarithmic branch cut at $E=0$.

Finally, another singularity of interest in the three-

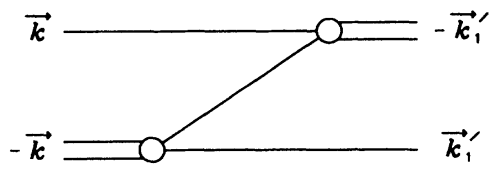

(a)

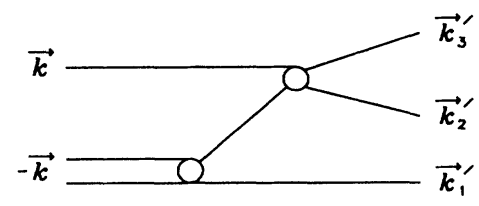

(b)

FIG. 2. Typical two-to-two and two-to-three amplitudes of lowest order. 
particle breakup process is the subenergy singularity. This is the singularity in the two-particle subenergy variable in the final state. This singularity is of relevance in the final-state interaction of the three-particle system and should be considered in breakup processes represented schematically as

$$
i+(j k) \rightarrow 1+2+3 .
$$

It will be treated in Sec. IV D.

In contrast to Sec. III, where we studied the analytic properties of the few-particle amplitudes from a consideration of the MS series, we base the study of analytic properties in this section on a consideration of various unitarity relations. Unitarity implies that scattering amplitudes should have singular behavior and an associated branch cut in the complex energy plane at various physical thresholds, with well-determined discontinuities. A knowledge of these discontinuities often allows one to predict the related singularities. Unitarity imposes specific constraints in the analytic behavior of different scattering amplitudes, which should be incorporated when modeling and parametrizing the scattering amplitudes. These constraints have been proved to be of relevance in three dimensions $[12,26]$.

\section{B. Singularity of the two-to-two amplitude at the $m$-particle threshold}

A discussion of these singularities in three dimensions has appeared in Refs. [12] and [20]. In general, the unitarity relation yields the contribution of each scattering threshold to the imaginary part of the scattering amplitude. We shall follow the diagrammatic representation of Refs. [12], [20], and [27] in multiparticle processes involving potentials.

We start this section by studying the behavior of twoto-two amplitude of the $m$-particle system as $E \rightarrow 0$. The singularity we are interested in comes from the unitarity relation shown schematically in Fig. 3, where the dashed vertical line represents on-shell propagation of $m$ particles given by the $\delta$-function part of the propagator, or the free $m$-particle Green's function, and the star represents complex conjugation. As we know that the amplitudes of Fig. 3 are finite as the total $m$-particle energy $E \rightarrow 0$, the contribution to the discontinuity of the two-to-two amplitude arising from the $m$-particle threshold is given by

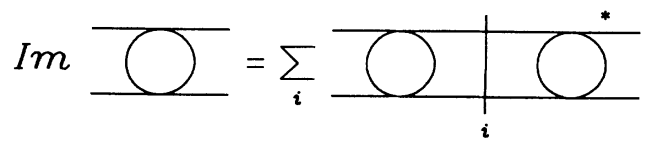

FIG. 3. Unitarity relation satisfied by the two-to-two amplitude of the $m$-particle system. The vertical line denotes free on-shell propagation in the intermediate state. The summation extends over the index $i$ which represents the number of particles in the intermediate state.

$$
\begin{aligned}
\mathcal{D}\left(T_{2 \rightarrow 2}\right) \propto \int & d \mathbf{k}_{1} d \mathbf{k}_{2} \cdots d \mathbf{k}_{m} \delta\left(E-k_{1}^{2}-k_{2}^{2}-\cdots-k_{m}^{2}\right) \\
& \times \delta\left(\mathbf{k}_{1}+\mathbf{k}_{2}+\cdots+\mathbf{k}_{m}\right)\left|T_{2 \rightarrow m}\right|^{2},
\end{aligned}
$$

where $\mathcal{D}$ denotes the discontinuity across the unitarity cut starting at the $m$-particle threshold, $\mathbf{k}_{i}$ is the momentum of particle $i$ in the intermediate state of Fig. 3, and $T_{2 \rightarrow m}$ is the $t$ matrix for the two-to- $m$ process.

The simplest case of Eq. (61) is given by $m=3$ in two-space dimensions. In this case

$$
\begin{aligned}
\mathcal{D}\left(T_{2 \rightarrow 2}\right) \propto \int & d^{2} k_{1} d^{2} k_{2} d^{2} k_{3} \delta\left(E-k_{1}^{2}-k_{2}^{2}-k_{3}^{2}\right) \\
& \times \delta\left(\mathbf{k}_{1}+\mathbf{k}_{2}+\mathbf{k}_{3}\right)\left|T_{2 \rightarrow 3}\right|^{2} .
\end{aligned}
$$

We would like to find the total three-particle energy dependence of $\mathcal{D}\left(T_{2 \rightarrow 2}\right)$, from which we can find the energy dependence of the singular part of $T_{2 \rightarrow 2}$. In order to find the energy dependence of expression (62), we define the hypermomentum $k$ by

$$
k^{2}=k_{1}^{2}+k_{2}^{2}+k_{3}^{2},
$$

in terms of which Eq. (62) can essentially be rewritten as

$$
\mathcal{D}\left(T_{2 \rightarrow 2}\right) \propto \int k^{3} d k d \Omega \delta\left(E-k^{2}\right)\left|T_{2 \rightarrow 3}\right|^{2},
$$

where $d \Omega$ is the angular integral subject to $\mathbf{k}_{1}+\mathbf{k}_{2}+\mathbf{k}_{3}=$ 0 . Remembering that at the three-particle threshold given by $E=0, T_{2 \rightarrow 3}$ tends to a constant, we have $\mathcal{D}\left(T_{2 \rightarrow 2}\right) \propto E$, which implies a singular part of the type

$$
\mathcal{S}\left(T_{2 \rightarrow 2}\right) \sim E \ln (-E)
$$

at the three-particle threshold. The two-to-two amplitude $T_{2 \rightarrow 2}$ is finite at the three-particle threshold $E=0$, but has a branch point there of the form $E \ln (-E)$.

The above idea can essentially be extended to the more complicated case of the $m$-particle threshold. For example, at the four-particle threshold,

$$
\begin{aligned}
\mathcal{D}\left(T_{2 \rightarrow 2}\right) \propto \int & d \mathbf{k}_{1} d \mathbf{k}_{2} d \mathbf{k}_{3} d \mathbf{k}_{4} \delta\left(E-k_{1}^{2}-k_{2}^{2}-k_{3}^{2}-k_{4}^{2}\right) \\
& \times \delta\left(\mathbf{k}_{1}+\mathbf{k}_{2}+\mathbf{k}_{3}+\mathbf{k}_{4}\right)\left|T_{2 \rightarrow 4}\right|^{2},
\end{aligned}
$$

where $E$.is now the four-particle c.m. energy. Again, in terms of the hypermomentum $k$ defined by $k^{2}=k_{1}^{2}+$ $k_{2}^{2}+k_{3}^{2}+k_{4}^{2}$, Eq. (66) can be rewritten as

$$
\mathcal{D}\left(T_{2 \rightarrow 2}\right) \propto \int k^{5} d k d \Omega \delta\left(E-k^{2}\right)\left|T_{2 \rightarrow 4}\right|^{2},
$$

where $d \Omega$ is the angular integral subject to $\mathbf{k}_{1}+\mathbf{k}_{\mathbf{2}}+\mathbf{k}_{\mathbf{3}}+$ $\mathbf{k}_{4}=0$. Remembering that at the four-particle threshold $E=0$ and $T_{2 \rightarrow 4}$ tends to a constant, we have

$$
\mathcal{D}\left(T_{2 \rightarrow 2}\right) \propto E^{2},
$$

which implies a singular part of the type

$$
\mathcal{S}\left(T_{2 \rightarrow 2}\right) \sim E^{2} \ln (-E)
$$

at the four-particle threshold. The variable $E$ in Eq. (65) 
is the total three-particle energy $(E=0$ is the threeparticle threshold); in Eq. (69), $E=0$ denotes the fourparticle threshold. The two-to-two amplitude $T_{2 \rightarrow 2}$ is finite at the four-particle threshold $E=0$, but has a branch point there of the form $E^{2} \ln (-E)$.

In general, the singular part of $T_{2 \rightarrow 2}$ at the $m$-particle threshold is given by

$$
\mathcal{S}\left(T_{2 \rightarrow 2}\right) \sim E^{m-2} \ln (-E) .
$$

\section{Singularity of the one-to-three and two-to-three amplitudes}

A discussion of these singularities in three dimensions has appeared in Refs. [12] and [28]. Again, we shall use relevant unitarity relations to find the discontinuity of the scattering amplitudes, from which we shall extract the singular parts. We follow the diagrammatic representation of Ref. [12], as we did in Sec IVB. The unitarity relation of interest for the two-to-three amplitude is shown diagrammatically in Fig. 4. The two-to-three amplitude has two types of singularities, given by the last two terms on the right-hand sides of Fig. 4. The second term on the right-hand-side of Fig. 4 involves the connected three-to-three amplitudes and contributes to certain singularities. The last term of Fig. 4 contributes to a subenergy singularity and depends on the momentum of particle $i$ in final states and is summed over $i$.

The first term on the right-hand side of Fig. 4 will contribute to the singularity in total three-particle c.m. energy $E$. The discontinuity we are interested in is given by

$$
\begin{aligned}
\mathcal{D}\left(T_{2 \rightarrow 3}\right) \propto \int & d \mathbf{k}_{1} d \mathbf{k}_{2} d \mathbf{k}_{3} \delta\left(E-k_{1}^{2}-k_{2}^{2}-k_{3}^{2}\right) \\
& \times \delta\left(\mathbf{k}_{1}+\mathbf{k}_{2}+\mathbf{k}_{3}\right) T_{2 \rightarrow 3} T_{3 \rightarrow 3}^{*} .
\end{aligned}
$$

We have seen in Sec. III that as $E \rightarrow 0$ the amplitude $T_{3 \rightarrow 3}$ of Eq. (71) is singular and divergent and is given by Eq. (46). The amplitude $T_{2 \rightarrow 3}$ is finite in this limit. The phase-space integral of Eq. (71), involving only the $\delta$ functions, will have the same behavior as in Eq. (62). Though the $t$ matrices $T_{2 \rightarrow 3}$ and $T_{3 \rightarrow 3}^{*}$, which appear on the right-hand side of Eq. (71), involve the momenta $\mathbf{k}_{1}, \mathbf{k}_{2}, \mathbf{k}_{3}$, in general, at low energies $(E \rightarrow 0)$, the energy-dependent singular part of $T_{3 \rightarrow 3}$ is given by Eq. (46) and in the same limit $T_{2 \rightarrow 3}$ tends to a constant. Hence in the $E \rightarrow 0$ limit the phase-space integral of Eq. (71) can be evaluated treating $T_{2 \rightarrow 3}$ and $T_{3 \rightarrow 3}$

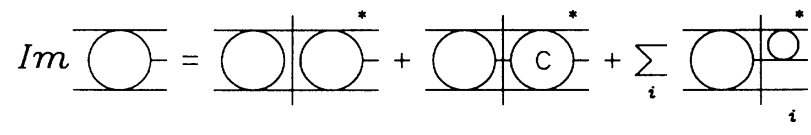

FIG. 4. Unitarity relation satisfied by the two-to-three amplitude of the three-particle system. The summation extends over $i$ which represents the noninteracting particle. Again the vertical line denotes free on-shell propagation in the intermediate state. as constants. We have seen in our discussion related to (62) that essentially the phase-space integral of Eq. (71) yields a singular part $E \ln (-E)$. This singularity, when multiplied by the low-energy behavior of $T_{3 \rightarrow 3}$ as given by Eq. (46), yields the following singular parts of $T_{2 \rightarrow 3}$ via Eq. (71): $\ln ^{-n}(-E), n=1,2,3, \ldots$. As powers of a logarithm yield a new singularity, we have an infinite number of branch points at $E=0$ in $T_{2 \rightarrow 3}$, e.g., $\ln ^{-n}(-E), n=1,2,3, \ldots$ By similar arguments, essentially this same singularity will appear in the one-to-three amplitude $T_{1 \rightarrow 3}$ in two dimensions [12]. This is of particular interest, as this amplitude is the time-reversed form of the three-to-one recombination amplitude $T_{3 \rightarrow 1}$ studied recently [5]. The three-to-one amplitude $T_{3 \rightarrow 1}$ in two dimensions will have the same singularities as $T_{1 \rightarrow 3}$.

\section{Subenergy singularity in the two-to-three amplitude}

The singularities in the subenergy variables in the breakup amplitude $T_{2 \rightarrow 3}$ are essential for a correct description of final-state interactions. Usually, in breakup processes, one measures the spectrum of a single particle. Then one requires the proper singularity structure of this amplitude as a function of the energy of this particle in order to make a correct parametrization. The nature of this singularity can be obtained from the last term of the unitarity relation in Fig. 4.

In Fig. 4 it is desirable to introduce the following momenta in the intermediate state of the last term. Let the particle labeled $i$ have momentum $\mathbf{p}$ and the other two particles have momenta $-\mathbf{p} / 2+\mathbf{q}$ and $-\mathbf{p} / 2-\mathbf{q}$. Then it is easy to see that the contribution to $\mathcal{D}\left(T_{2 \rightarrow 3}\right)$ from the last term of Fig. 4 is given by

$\mathcal{D}\left(T_{2 \rightarrow 3}^{(i)}\right) \sim \sum_{\substack{i, j \\ j \neq i}} \int d \mathbf{q} \delta\left(E-\frac{3}{2} p^{2}-q^{2}\right) T_{2 \rightarrow 3}^{(j)} t_{j}^{*}\left(q^{2}\right)$,

where the energy argument of the two-particle $t$ matrix $t_{j} \equiv t_{i k}, i \neq j \neq k \neq i$, is explicitly shown. Equation (25) when substituted into Eq. (72) yields the following complicated expression for the discontinuity of $T_{2 \rightarrow 3}$ in the branch cut in subenergy:

$$
\begin{aligned}
\mathcal{D}\left(T_{2 \rightarrow 3}\right) \sim & {\left[-\frac{2}{\ln \left(-E+3 p^{2} / 2\right)}\right.} \\
& \left.+\frac{2 b}{\ln ^{2}\left(-E+3 p^{2} / 2\right)}-\cdots\right]
\end{aligned}
$$

and it is difficult to write an exact form for this singularity.

The singularity of Eq. (73) lies at the boundary of the physical region because the allowed values of $p^{2}$ are from 0 to $2 E / 3$. This singularity leads to rapid variations in the final-state amplitude and is crucial to the phenomenological treatment of the final-state interaction. 


\section{SUMMARY}

We have presented a discussion of analytic behavior in the complex energy plane of the various few-particle scattering amplitudes in two dimensions. A knowledge of these analytic behaviors is relevant for a complete understanding of different few-particle processes. We have based our discussion on distinct methods for different amplitudes. A consideration of the full set of dynamical equations for the few-particle system $[12,29]$ should always reveal these analytic properties. However, this approach is not always the most convenient one. Depending on a specific amplitude, we have adopted a specific method.

In Sec. II we based our discussion of the low-energy on- and off-shell two-particle $t$ matrix on the dynamical equations. We showed that in the low-energy-momentum limit both the on- and the off-shell two-particle $t$ matrices tend to zero and have an infinite number of logarithmic branch points at $E=0$.

In Sec. III we based our discussion of the low-energy behavior of the connected three-particle (three-to-three) amplitude on a consideration of the MS series. We showed that in the low-energy limit this amplitude is divergent and possesses an infinite number of logarithmic branch points at $E=0$. We concluded that the connected $m$-particle amplitude also diverges in this limit and possesses similar branch points.

In Sec. IV we based our discussion of the low-energy behavior of the two-to-three and the two-to-two amplitudes on a consideration of unitarity. The constraints of unitarity impose specific analytic properties on these amplitudes. We showed that, unlike the case of the three-tothree amplitude, these amplitudes are finite in this limit. However, these amplitudes possess specific logarithmic singularities.

\section{ACKNOWLEDGMENT}

The work is supported in part by the Conselho Nacional de Desenvolvimento-Científico e Tecnológico (CNPq) of Brasil.
[1] M. L. Cramer, L. W. Bruch, and F. Cabral, J. Chem. Phys. 67, 1442 (1977), L. W. Bruch and J. A. Tjon, Phys. Rev. A 19, 425 (1979), F. Cabral and L. W. Bruch, J. Chem. Phys. 70, 4669 (1979).

[2] J. A. Tjon, Phys. Rev. A 21, 1334 (1980).

[3] L. P. H. de Goey, J. P. J. Driessen, B. J. Verhaar, and J. T. M. Walraven, Phys. Rev. Lett. 53, 1919 (1984).

[4] S. K. Adhikari, A. Delfino, T. Frederico, I. D. Goldman, and L. Tomio, Phys. Rev. A 37, 3666 (1988).

[5] C. T. Christou and M. I. Haftel, Few-Body Systems 9, 195 (1990); 7, 1 (1989); M. I. Haftel and T. K. Lim, J. Chem. Phys. 84, 4407 (1986).

[6] L. P. H. de Goey, T. H. M. van der Berg, N. Mulders, H. T. C. Stoof, B. J. Verhaar, and W. Glöckle, Phys. Rev. B 34, 6183 (1986); L. P. H. de Goey et al., ibid. 38, 646 (1988); 38, 1150 (1988).

[7] H. F. Hess et al., Phys. Rev. Lett. 51, 483 (1983); 52, 1520 (1984).

[8] R. Sprik, J. T. M. Walraven, and I. F. Silvera, Phys. Rev. Lett. 51, 479 (1983); Phys. Rev. B 32, 5668 (1985).

[9] A. Lagendijk, Phys. Rev. B 25, 2054 (1982); E. V. L. Mello, J. J. Rehr, and O. E. Vilches, ibid. 28, 3859 (1983).

[10] J. G. Dash, Phys. Rep. 38C, 177 (1978).

[11] M. V. N. Murthy, J. Law, M. Brack, and R. K. Bhaduri, Phys. Rev. Lett. 67, 1817 (1991); M. Sporre, J. J. M. Verbaarschot, and I. Zahed, ibid. 67, 1813 (1991); A. Suzuki, M. K. Srivastava, R. K. Bhaduri, and J. Law, Phys. Rev. B 44, 10731 (1991); R. K. Bhaduri, R. S. Bhalerao, A. Khare, J. Law, and M. V. N. Murthy, ibid. 66, 523 (1991); A. Comtet, J. McCabe, and S. Ouvry, Phys. Lett. B 260, 372 (1991).

[12] S. K. Adhikari and K. L. Kowalski, Dynamical Collision Theory and Its Applications (Academic, Boston, 1991), Chap. 6.

[13] L. H. Thomas, Phys. Rev. 47, 903 (1935); V. Efimov, Phys. Lett. 33B, 563 (1970).
[14] S. K. Adhikari and R. D. Amado, Phys. Rev. Lett. 27, 485 (1971).

[15] W. G. Gibson, Phys. Rev. A 6, 2469 (1972).

[16] R. L. Siddon and M. Schick, Phys. Rev. A 9, 907 (1974); W. G. Gibson, Mol. Phys. 49, 103 (1983); Phys. Rev. A 36, 564 (1987).

[17] R. G. Newton, Scattering Theory of Waves and Particles, 2nd ed. (Springer-Verlag, New York, 1982).

[18] M. G. Fuda, Phys. Rev. C 5, 275 (1972); see, in particular, Ref. [15], Appendix C.

[19] R. D. Amado and M. Rubin, Phys. Rev. Lett. 25, 194 (1970); S. K. Adhikari, Phys. Rev. D 8, 1195 (1973).

[20] P. R. Graves-Morris, Ann. Phys. (N.Y.) 41, 477 (1967).

[21] S. K. Adhikari, Am. J. Phys. 54, 362 (1986).

[22] P. M. Morse and H. Feshbach, Methods of Theoretical Physics (McGraw-Hill, New York, 1953), Chap. 11.

[23] D. Bolle and F. Gesztesy, Phys. Rev. Lett. 52, 1469 (1984); Phys. Rev. A 30, 1279 (1984).

[24] B. J. Verhaar, J. P. H. W. van den Eijnde, and M. A. J. Schaffrath, J. Phys. A 17, 595 (1984).

[25] S. K. Adhikari, W. G. Gibson, and T. K. Lim, J. Chem. Phys. 85, 5580 (1986).

[26] R. R. Aaron and R. D. Amado, Phys. Rev. Lett. 31, 1157 (1973).

[27] R. J. Eden, P. V. Landshoff, D. I. Olive, and J. C. Polkinghorne, The Analytic $S$-Matrix (Cambridge University Press, Cambridge, 1966).

[28] R. D. Amado, D. F. Freeman, and M. H. Rubin, Phys. Rev. D 4, 1032 (1971).

[29] L. D. Faddeev, Mathematical Aspects of the Three-Body Problem in Quantum Scattering Theory (Davey, New York, 1965); R. D. Amado, in Elementary Particle Physics and Scattering Theory, edited by M. Chretien and S. S. Schweber (Gordon and Breach, New York, 1970), Vol. 2, p. 1; C. Lovelace, Phys. Rev. 135, B1225 (1964); A. N. Mitra, Adv. Nucl. Phys. 3, 1 (1969). 